\section{RECUERDO Y COMUNICACIÓN: SOCIO-HERMENÉUTICA DE RITUALES DE MEMORIA}

\author{
Bernt Schnettler y Alejandro Baer \\ Universität Bayreuth \\ schnettler@uni-bayreuth.de
}

\section{REMEMBRANCE AND COMMUNICATION: SOCIO- HERMENEUTICS OF MEMORY RITUALS}

Cómo citar este artículo/ Citation: Schnettler, B. y Baer, A. (2013). "Remembrance and communication: socio-hermeneutics of memory rituals". Arbor, 189 (761): a041. doi: http://dx.doi. org/10.3989/arbor.2013.761n3009

Recibido: 15 abril 2011. Aceptado: 25 octubre 2012

RESUMEN: El análisis de rituales performativos de memoria supone un reto metodológico para la investigación social interpretativa, ya que las interacciones rituales están inscritas en un contexto más amplio, que está determinado por las tradiciones simbólicas y por los acuerdos institucionales que configuran el marco de las ceremonias. A partir del estudio de ceremonias conmemorativas del Día Internacional de Recuerdo del Holocausto se mostrará la interconexión en el análisis de estos distintos niveles. Para ello nos basamos en datos videográficos de un proyecto de investigación en curso sobre ceremonias de memoria del Holocausto en Espana y Alemania, que examina la tesis sobre una incipiente "cultura de la memoria global". En el análisis de los materiales seguimos enfoques metodológicos que provienen de la sociología del conocimiento hermenéutica y la sociología de géneros comunicativos.

PALABRAS CLAVE: Memoria social; sociología del conocimiento; rituales; video-análisis; etnografía focalizada; Holocausto.
Copyright: (C) 2013 CSIC. This is an open-access article distributed under the terms of the Creative Commons Attribution-Non Commercial (by-nc) Spain 3.0 License.
ABSTRACT: The analysis of memory rituals represents a methodological challenge for an interpretative social science. Ritual interactions are inscribed in a broader context, which is determined by symbolic traditions and the institutional agreements that configure the general framework of ceremonies. The study of rituals held on International Holocaust Remembrance Day on January 27th is an opportunity to highlight the linkages between these different levels in the analysis. Our data is based on a videographic research project that probes into the thesis of a "global" memory culture, according to which particular memories coexist and the same time converge and interact with transnational forms of remembrance. For the analysis we follow methodological approaches rooted in the sociology of knowledge and the sociology of communicative genres.

KEYWORDS: Social memory; Sociology of knowledge; rituals; video analysis; focused Ethnography; Holocaust.

Este texto se basa en una conferencia presentada en el Congreso Alemán de Sociología que tuvo lugar en Jena en 2008. Una versión anterior está incluida en las actas del congreso: Bernt Schnettler, Alejandro Baer, Dariuš Zifonun: "Transnationale Gedächtniskultur? Ansätze einer ländervergleichenden Performanzanalyse von Erinnerungsritualen am Beispiel des 27. Januars", in: Soeffner, HansGeorg (Ed.), Unsichere Zeiten. Herausforderungen gesellschaftlicher Transformationen. Verhandlungen des 34. Kongresses der Deutschen Gesellschaft für Soziologie in Jena 2008, Wiesbaden: VS-Verlag, 2010 (CD-Rom) 


\section{EINLEITUNG: TRANSNATIONALISIERUNG DER ERINNERUNGSKULTUR?}

In der Erinnerungsforschung wird gegenwärtig die These der Ausbildung einer übernationalen Erinnerungskultur diskutiert. Für die Beantwortung der Frage nach einer möglichen Konvergenz von partikularen Erinnerungskulturen sind geeignete Vergleiche erforderlich. Der Beitrag skizziert die Richtung und Arbeitsweise einer darauf ausgerichteten empirischen Untersuchung. Ihr Gegenstand sind die jährlich am 27. Januar stattfindenden Veranstaltungen zum Internationalen Holocaust-Gedenktag, anhand deren sich exemplarisch die Formen öffentlich performierter, transnationalstaatlich orientierter Erinnerungspolitik studieren lassen. Methodisch werden dabei videoanalytische und ethnografische Mittel eingesetzt. Im Fokus der Forschung steht die sorgfältige Untersuchung des Ablaufs der zentralen offiziellen Gedenkveranstaltungen in Deutschland und Spanien sowie deren Einbettung und Wirkung im jeweiligen ,Feld der Erinnerung' in beiden Ländern. Auf der Basis ausführlicher Analysen einer sich in öffentlichen Veranstaltungen präsentierenden Erinnerungsform zielt die Untersuchung auf einen Beitrag zur Erhellung der Erinnerungskultur im Spannungsfeld zwischen angestrebter Globalisierung des Gedächtnisses einerseits und der Notwendigkeit zur markierten Darstellung partikularer Anliegen und symbolischer Bestände einzelner Kollektive andererseits. Weil sich die Untersuchung noch in einer frühen Phase befindet, können hier zwar noch keine Ergebnisse vorgestellt werden. Es sollen jedoch zumindest der Ansatz und die inhaltliche Stoßrichtung der Forschung skizziert werden.

Für die Herstellung neuer, sinnverbindender und einheitsstiftender sozialer Ordnungen, welche die engen Grenzen nationalstaatlicher Räume zu überschreiten trachten, wird Kollektivritualen ein besonderer Wert beigemessen. Mitunter werden sogar neue Zeremonien dieser Art mit der ausdrücklichen Absicht ins Leben gerufen, die faktisch noch nicht oder nur unvollkommen gegebene, aber gewollte Einheit, die sie vorgeblich repräsentieren, überhaupt erst zu stiften. In einer durch grenzüberschreitende Mobilität und Massenmedien gebildeten ,neuen Weltordnung' ergeben sich dabei allerdings bislang unbekannte symbolische Komplexitäten und Aporien. Wie Soeffner (2000: 256) hervorhebt, gilt diese Widersprüchlichkeit prinzipiell schon für die Innenbetrachtung aller, fortgeschrittenen' Gesellschaften: „Während geschlossene Gesellschaften sich auf eine konsistente symbolische Ordnung stützen können, werden offene Gesellschaften mitgeformt vom Wettkampf der Symbole“. Mit begründeter Skepsis begegnet er der seinem Verständnis nach fruchtlosen Hoffnung, Kollektivritualen in fortgeschritten modernisierten, ,offenen' Gesellschaften könne gemeinschaftsstiftende Kraft zugemessen werden, welche bestehende Mehrdeutigkeiten, Widersprüchlichkeiten und Interessenantagonismen überwinden helfe und - gewissermaßen durch den rituellen Vollzug selbst - die herbeigesehnte neue Einheit erzeugt. Fraglos bedürfen alle Kollektive einer Zelebrierung periodisch wiederkehrender Rituale der Selbstvergewisserung. Erinnerungsrituale verweisen deshalb immer auf einen doppelten Horizont, indem sie im gegenwärtigen Vollzug eine Version gemeinsam vorgestellter Vergangenheit in den Dienst eines bestimmten, für das jeweilige Kollektiv bedeutsamen Zukunftsprojektes stellen. Sie sind stark von regionalen oder nationalen Präferenzmustern sowie vom historischen Erbe der jeweiligen Gruppe mitgeprägt und gestalten sich in einem breiten Spektrum, das von ,heroisierenden' und triumphalistischen Gedenkformen bis zu dem nicht allein dem vermeintlichen deutschen Sonderfall vorbehaltenen ,traumatischen Erinnern als kollektivbegründender Ressource reicht. Lamentos, Freudentaumel oder getragene Ernsthaftigkeit mögen dem jeweiligen Gedenkritual eine ganz unterschiedliche emotionale Grundgestimmtheit unterlegen. In der Hoffnung auf ihre jeweilige verbundenheitserzeugende Wirkung können jedoch auch höchst unterschiedlich ausfallende Veranstaltungen in ihren vermeinten Zielen konvergieren.

Ebenso ist es keineswegs historisch neu, dass Kollektivrituale mit der ausdrücklichen Absicht erfunden und ausgestaltet werden, eine bestehende Erinnerungsgemeinschaft durch absichtsvolle Überschreitung zu erweitern bzw. eine noch zu formende, erweiterte Gemeinschaft unter dem symbolischen Dach eines neuen Rituals hervorzubringen. Wie die Untersuchungen zu den Olympischen Spielen der Neuzeit von MacAlloon (2009) zeigen, sind Ritualdirigenten und -organisatoren dabei vor allem durch die mitunter unwillentlich und unabsichtlich in Konkurrenz tretenden divergierenden Deutungen verwendeter Symbolelemente aus der Perspektive der verschiedenen, kleineren ,Ursprungskollektive' herausgefordert, die das Ritual zu einer größeren Einheit zu verschmelzen trachtet.

Ein solches Projekt moralisch fundierter Selbsterzeugung eines neuen, Partikulargemeinschaften überschreitenden , moralischen Kollektivs' ist der Internationale Holocaust-Gedenktag. Seine Leitintention zielt 
programmatisch auf eine, Globalisierung der Erinnerung' und nimmt seinen Ausgang beim Holocaust als historischem Paradigma dessen, was die absolute Grenze des Menschlichen markiert. Der HolocaustGedenktag ist allerdings kein Trauerritual - und in das Schema von Triumpf oder Trauma (Giesen 2004) ließe er sich nur mit Mühen zwängen. Der Absicht nach transzendiert er das Gedenken einer partikularen Opfergruppe und wird als Instrument einer prinzipiell inkludierenden, auf Humanität allgemein zielenden übernationalen Gemeinschaft etabliert, die vor allem im Prozess der Integration der europäischen Staaten zu einem transnationalen Verbund eine bedeutsame Rolle spielt (Leggewie 2009). Die - bislang - geringe öffentliche Bekanntheit des Internationalen HolocaustGedenktags steht der Absicht nicht entgegen, ganz zweifellos ein Instrument moralischer Politik zu sein.

Für unsere Fragestellung sind vor allem kulturvergleichende Aspekte relevant. Deshalb führen wir derzeit eine Studie durch, die im Schnittpunkt kultursoziologischer, ritualtheoretischer sowie kommunikationsanalytischer Forschung angesiedelt ist und auf einen Vergleich der Erinnerungskulturen Spaniens und Deutschlands zielt. Als Vergleichsobjekt bietet der am 27. Januar zelebrierte Internationale HolocaustGedenktag den Vorzug, Erinnerungskulturen in unterschiedlichen nationalkulturellen Kontexten fokussiert und kontrastierend untersuchen zu können. Mit der Forschung soll bestimmt werden, ob sich Konvergenzen einer transnationalen Gedächtniskultur entwickeln und worin diese ggf. bestehen. Im Mittelpunkt der Untersuchung steht die detaillierte videoanalytische Rekonstruktion, Analyse und Interpretation der zentralen Gedenkveranstaltungen zum 27. Januar, wobei vor allem deren performativer Ablauf und die eingesetzten Symboliken untersucht werden. Ergänzt wird dies durch fokussierte ethnografische Feldstudien, die darauf zielen, die zivilgesellschaftlichen und institutionellen Kontexte des Feldes der Erinnerung zu erheben, in welche diese Gedenkveranstaltungen eingebettet sind. Damit soll der, Sitz im gesellschaftlichen Leben' der offiziellen Gedenkakte der beiden Länder bestimmt und Aussagen über deren Kulturbedeutsamkeit ermöglicht werden.

\section{ERINNERUNGSFORSCHUNG}

Die Beschäftigung mit Fragen des ,kollektiven Gedächtnisses' hat sich über die vergangenen Jahre hinweg in den Sozial- und Kulturwissenschaften intensiviert. Nicht nur in Deutschland nimmt dabei die Erinnerung an die nationalsozialistischen Verbrechen eine gleichsam paradigmatische Rolle ein. Die bisherige Forschung zu kollektiven Erinnerungsphänomenen lässt sich entlang einer von Assmann (1997) eingeführten Unterscheidung gruppieren. Assmann differenziert bekanntlich zwischen dem alltagsnahen, kurzzeitigen und auf persönlicher Interaktion begründeten kommunikativen Gedächtnis einerseits und dem auf dauerhafte Fixierung zielenden kulturellen Gedächtnis bzw. der institutionalisierten, öffentlichen Erinnerungskultur andererseits. Diese idealtypische Trennung zwischen Gedächtnisformen ist zunächst der theoretischen Klärung dienlich. Als analytisch besonders fruchtbar haben sich Forschungsansätze erwiesen, die empirisch wie konzeptionell an der Nahtstelle zwischen kommunikativem und kulturellem Gedächtnis ansetzen. In diesem Sinne zielen eine Reihe von Studien auf die Analyse des Ausprägungsprozesses kultureller Erinnerungsformen in kommunikativen Akten und die oftmals konflikthaften Verständigungsprozesse über die Institutionalisierung kollektiver Symbole, Rituale und Erinnerungsdiskurse.

Vier Themenfelder lassen sich hier identifizieren: An erster Stelle stehen eine Fülle von Arbeiten über Erinnerungsorte (Zifonun 2004). Die Menge der wissenschaftlichen Studien über Erinnerungsmale ist annähernd so groß wie die Zahl der Monumente, die unseren öffentlichen Raum bevölkern. An zweiter Stelle stehen Gedenktage und Erinnerungsjahre als Erinnerungsmale in der Zeit. Sie bieten Gelegenheit, vergangene Ereignisse performativ zu vergegenwärtigen. Im rituellen Vollzug symbolträchtiger Handlungen weisen zyklische Erinnerungsveranstaltungen einen starken Ereignischarakter aus: in ihnen wird Erinnerung zeitlich verdichtet zum erfahrungsträchtigen Event (Nora 1974; Sahlins 1991), das den einzelnen im Handlungsvollzug des Gedenkens mit dem Kollektiv zusammenschließt. An dritter Stelle sind verschiedene (massen-)mediale Formen der Erinnerung zu nennen (Baer 2005). In spätmodernen, von Technologie und Medien umfassend durchwirkten Gesellschaften wird die Erinnerungskultur durch medial vermittelte Elitendebatten stark mitgeprägt. Medien ermöglichen nicht allein eine Erinnerungsexpansion, indem sie über die Anwesenden hinaus eine - wenngleich mittelbare Beteiligung für Abwesende erlauben. Zugleich führen sie auch die Gefahr mit sich, dass öffentliches Erinnern - zumindest der Tendenz nach - zu reinem Spektakel und zur Unterhaltung zu verkommen und damit seine für die Moderne typischen Funktionen von Identitätserhalt und Tradierung zu verlieren droht. Schließlich existiert viertens ein wachsendes Forschungsinteresse an der Geschichts- und Erinnerungspolitik. Die Art 
und Weise der Erinnerung an historische Ereignisse ist gesellschaftlich häufig umstritten. Erinnerungskultur ist dann zugleich Erinnerungspolitik (Soeffner 2006), wenn man sich der Frage nach den gesellschaftlichen Auseinandersetzungen um die Durchsetzung von Geschichtsdeutungen sowie den Konflikten um die Bedeutung von Orten und Ereignissen zuwendet.

Die Forschungslage zu den vorgenannten Bereichen weist klare Schwerpunktsetzungen auf: Zu allen vier Forschungsfeldern liegt bereits eine ganze Reihe von Studien vor. Dabei nehmen die Untersuchungen zu Gedenkstätten einen prominenten Platz ein. Studien über Gedenktage und Erinnerungsjahre sind im Vergleich dazu selten. Medienanalysen finden sich hingegen in großem Umfang und zu zahlreichen Details (etwa über die ,Bitburg-Affäre', ,Brandts Kniefall', den so genannten Historikerstreit, die Walser-Bubis-Debatte usw.) und ebenso liegt eine Reihe von Arbeiten aus dem Bereich der Geschichts- und Erinnerungspolitik vor.

In Ergänzung dieser vier Themenfelder der Erinnerungsforschung ist als fünftes in den letzten Jahren in vergleichender Perspektive vermehrt die Frage nach der Entstehung einer, europäischen Erinnerungskultur' aufgeworfen worden (Lenz et al. 2002; Flacke 2004). Dabei spielt der Holocaust als paradigmatischer Erinnerungsgegenstand eine an Bedeutung wachsende Rolle in einer Entwicklungstendenz, die von nationaler, bislang staatsoffizieller Institutionalisierung zu einer möglichen post-nationalen Erinnerung überleitet (Gillis 1994). Letztere ist durch Pluralisierung gekennzeichnet, indem bislang ,vergessene' Opfergruppen in die erweiterte Erinnerungsgemeinschaft eingeschlossen werden, und wirft transnationale Gemeinsamkeiten auf. Françoise (2004) erkennt einen Prozess der Pluralisierung der Gedenkkulturen, der von der Basis ausgehend eine länderübergreifende Bewegung umfasst und in eine Lücke vorstößt, in der Staaten zurückhaltend bleiben oder erst spät reagieren. In diesem Prozess bilden sich neue Formen der Erinnerung aus, die sich etwa in Gedenkstätten, Holocaust-Museen oder der Einführung von Gedenktagen äußert, die von den heroischen Meistererzählungen von Heldentum und Widerstand abweichen und unter dem Zeichen des nazistischen Völkermords stehen. Diese Entwicklungen können, so Françoise, als „zentrale Bestandteile einer entstehenden europäischen bzw. westlichen Öffentlichkeit und Gedächtniskultur betrachtet werden". (Françoise 2004: 23) In diesem Zusammenhang ist auch die These einer globalisierten Holocausterinnerung formuliert wor- den, die sich in einem Gedenken jenseits der Nation und einem von unmittelbaren Opfergruppen gelösten Vergangenheitsbezug auf die nationalsozialistischen Verbrechen ausdrückt (Levy/Sznaider 2001; Alexander 2002). Die ,Kosmopolitisierung des Holocaustgedächtnisses' überschreite den europäischen Rahmen, weil sie aufgrund gemeinsam erinnerter Barbarei die Ausbildung einer globalen Gedächtniskultur stifte ohne jedoch zwangsläufig nationale Erinnerungen zu ersetzen. Diese These postuliert, aus dem inzwischen zu einem Symbol des absolut Bösen avancierten Holocaust würden globale Werte abgeleitet, die in Form von ,avoidance imperatives' transnationale konsensstiftende Zivilisationsstandards (Dubiel 2003) begründeten. Kritische Einschätzungen vermeinen gar, die mögliche Ausbildung einer neuen ,Zivilreligion' des Okzidents zu konstatieren (Probst 2002; Traverso 2007). Im Zuge dieser Entwicklung kämen auch Gesellschaften und Gruppen ohne unmittelbaren geografischen, historischen oder ethnischen Bezug zum Holocaust mit dessen symbolischer Bedeutung und sozialen Wirkung in Berührung.

So weitreichend und bedeutsam die These einer möglichen Kosmopolitisierung des Gedächtnisses ist, so erstaunlich dürftig fallen die dazu bisher vorliegenden empirischen Befunde aus. Die Antwort auf die Frage, wie sich diese entstehende europäische bzw. globale Holocausterinnerung als Wissens- und Kulturphänomen über Medien, Denkmäler, Gedenkstätten und -zeremonien in Erinnerungsdiskurse ausprägt, weist in den vorhandenen Arbeiten zahlreiche Lücken auf.

Wie Connerton in seinem Grundlagenwerk "How Societies Remember" (1989) formuliert, fällt Erinnerungszeremonien in diesem Zusammenhang eine besondere Rolle zu: "If there is such a thing as social memory, we are likely to find it in commemorative ceremonies" (Connerton 1989: 4). Gedenkrituale begründen als symbolisch-performative Praxis eine wesentliche Konstruktionsweise kollektiver Selbstbilder (Buser/Rauer 2004). Insofern stellt die Analyse des internationalen Holocaust-Gedenktags ein bedeutsames Forschungsdesiderat dar, weil sich an diesem Beispiel die oben erwähnten Defizite der bisherigen Erinnerungsforschung im Kontext einer globalisierten Erinnerungskultur exemplarisch und in kondensierter Form studieren lassen. Die Fixierung eines - nationalstaatliche Bezüge explizit übergreifenden - Gedenktags erlaubt es, die bislang stark vernachlässigte kulturvergleichende Forschung konzentriert voranzutreiben. Eine derartige Analyse lässt 
empirisch fundierte Ergebnisse erwarten, welche eine begründete Beurteilung der These des Entstehens einer globalisierten Erinnerungskultur bzw. einer Kosmopolitisierung des Gedenkens erwarten lassen. Diese Fokussierung ist nicht nur die Voraussetzung einer bewältigbaren Forschungsaufgabe im überaus ausgedehnten Feld der Erinnerungskulturen, die sich aufgrund ihrer häufigen nationalen und regionalen Spezifika zudem einer schlichten Vergleichsgrundlage entziehen. Die Konzentration auf ein exemplarisches, jährlich wiederholtes Gedenkereignis erleichtert außerdem in seiner simultanen Betrachtung in den verschiedenen nationalen Kontexten deswegen seine Vergleichbarkeit, weil es aufgrund seiner Anlage auf die gleichzeitige Realisierung in verschiedenen Kulturen hin angelegt ist. Seine tatsächliche Ausgestaltung in den einzelnen Kontexten ist hingegen die offene Frage, die Gegenstand einer empirischen Untersuchung sein muss.

\section{DER 27. JANUAR}

Die Hintergründe der Einrichtung des 27. Januars als Internationalem Holocaust-Gedenktag müssen kurz rekapituliert werden: Die verspätete Auseinandersetzung mit verschiedenen Formen von Kollaboration oder Mitverantwortung für die Naziverbrechen hat dazu beigetragen, dass über die beteiligten und betroffenen Staaten hinaus auch in anderen europäischen Ländern der Holocaust zum Bezugspunkt und Gestaltungsmoment öffentlicher Erinnerungspolitik wurde. Des Weiteren hat der erwähnte universelle moralpolitische Anspruch des Holocausts zur Einführung neuer Formen gesellschaftlichen und öffentlichen Gedenkens beigetragen, mit eigenen Veranstaltungen wie Lesungen, Schweigeminuten, Vorträgen und Filmvorführungen, die speziell an die Verfolgung und deren Opfer erinnern. Nach dem Muster des Holocaust-Gedenktags in Israel (Yom Hashoah) haben die meisten Länder Europas in der Folge neue Gedenktage eingeführt, die an die Deportation und Ermordung der Juden oder an die Befreiung des Konzentrationslagers Auschwitz erinnern.

Yom HaShoah Ve-Hagevurah heißt wörtlich ,Tag (Yom) zum Gedenken des Holocaust (Shoah) und des Heldentums (Gevurah)' und beginnt nach hebräischem Kalender bei Sonnenuntergang am 27. Tag des Monats Nissan. Er fällt in der Regel in den März oder April des gregorianischen Kalenders. Yom HaShoah wurde von der israelischen Regierung 1951 zum offiziellen Gedenktag etabliert. Im ganzen Land ertönen zwei Minuten lang Sirenen zu Ehren der ermordeten Juden. Vergnügungsstätten wie Theater, Tanzlokale, Restaurants und Cafés sind an diesem Tag geschlossen. Dieser Tag wird von den meisten jüdischen Gemeinschaften in der ganzen Welt begangen, obwohl orthodoxe Juden des Holocausts am 9. Av gedenken, dem Jahrestag der Zerstörung des Jerusalemer Tempels, an dem das Judentum die Erinnerung an die schrecklichsten Zerstörungen rituell begeht.

Die Einrichtung des 27. Januars als Internationalem Holocaust-Gedenktag wurde institutionell durch verschiedene internationale Konferenzen und $\mathrm{Ab}$ -

Abbildung 1. Holocaustgedenken in Europa

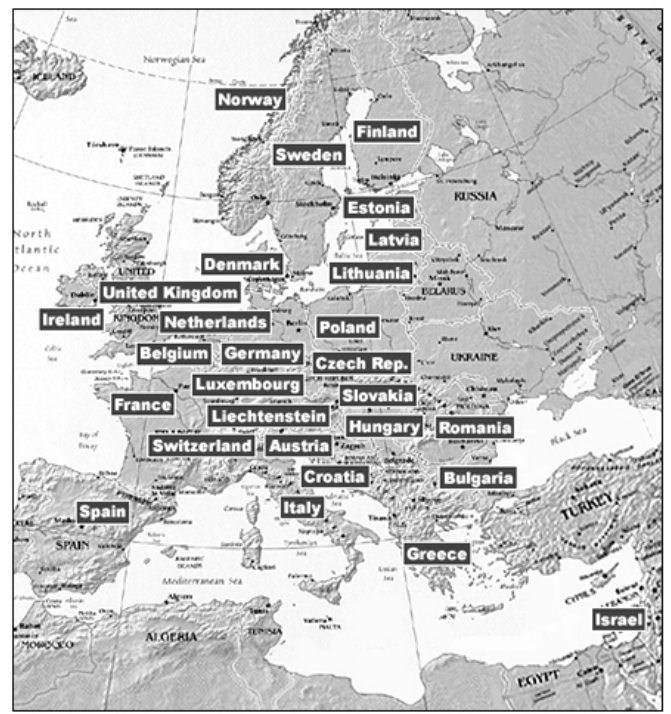

Quelle: http://www1.yadvashem.org/education/ceremonies/liberation/map/map.html 
kommen ermöglicht, bei der die 1998 von den Regierungen Großbritanniens, der USA und Schwedens ins Leben gerufene ,Task Force for International Cooperation on Holocaust Education, Remembrance and Research' eine zentrale Rolle spielte. Das erste Resultat der ,Task Force' war das Zusammentreffen von 47 Regierungschefs und Staatsvertretern beim ,Stockholm International Forum on the Holocaust' im Januar 2000. Das offizielle Ziel der Veranstaltung, an der auch eine beträchtliche Anzahl von Diplomaten, Vertretern von NGOs, religiösen Führern, Holocaust-Überlebenden, Akademikern, Lehrern und Journalisten teilnahmen, bestand darin, im internationalen Dialog "Anstrengungen zur Förderung der Aufklärung, des Erinnerns und der Forschung im Bereich des Holocaust zu verstärken" (Stockholm Declaration ${ }^{1}$ ). Das unmittelbare Ergebnis des Forums war die Verständigung auf Einführung eines jährlichen durchzuführenden internationalen Holocaust-Gedenktags am 27. Januar, dem Datum der Befreiung des Vernichtungslagers Auschwitz. Im Oktober 2002 verabschiedeten die Bildungsminister der Mitgliedsstaaten des Europarats eine Resolution, wonach in allen Schulen der betreffenden Länder ein Gedenktag zur Erinnerung an den Holocaust eingeführt werden soll. Bis zum heutigen Tage haben 18 von 55 OSZE-Mitgliedsstaaten den 27. Januar zum nationalen Holocaust-Gedenktag erklärt. 13 Staaten haben ein anderes Datum gewählt, das in engem $\mathrm{Zu}$ sammenhang zu ihrer nationalen Geschichte steht. In sechs OSZE-Staaten wird das Gedenken an die Holocaust-Opfer in einen nationalen Gedenktag integriert, der aber kein spezifischer Tag zur Erinnerung an die Opfer des Holocaust ist (siehe Abb. 1 sowie die Materialien unter dem angegebenen Link). Während der 60. Vollversammlung beschlossen die Vereinten Nationen im November 2005, den 27. Januar zum internationalen Tag des Gedenkens an die Opfer des Holocaust zu erklären. Die Schaffung dieses übernationalen kollektiven Gedenkdatums hat entscheidenden Anteil an der Ausbildung einer europäischen Identität und wirkt gleichsam als Integrationskatalysator in eine europäische Wertegemeinschaft: „Die Partnerschaftsprogramme der Task Force haben dazu beigetragen, ein gemeinsames Verständnis von europäischen Werten zu schaffen und den EU-Neulingen aus Ost- und Mitteleuropa und darüber hinaus bis in die Ukraine zu vermitteln" (Østergård 2008: 27).

\section{HOLOCAUSTGEDENKEN IN DEUTSCHLAND UND SPANIEN}

Gleichwohl erfolgt die Etablierung in den einzelnen Ländern höchst unterschiedlich. So wurde der 27. Januar in Deutschland bereits 1996 auf Initiative von Bundespräsident Roman Herzog als, Tag des Geden- kens an die Opfer des Nationalsozialismus' eingerichtet - und damit vor seiner internationalen Institutionalisierung als ,Holocaust-Gedenktag'. Im Gegensatz dazu wurde der 27. Januar in Spanien im internationalen Vergleich erst spät, nämlich erstmals offiziell im Jahr 2005 als ,Tag des Gedenkens an den Holocaust und Verhinderung der Verbrechen gegen die Menschlichkeit' begangen.

Unsere vergleichende Untersuchung beider Veranstaltungen soll Licht auf die Frage werfen, ob sich in diesem Zuge Indizien für die Herausbildung einer europäischen Erinnerungskultur finden lassen. Aus diesem Grund werden aus dem sehr viel breiteren internationalen Feld diese zwei einander maximal kontrastierenden Fälle ausgewählt: Der Ländervergleich Spanien-Deutschland zum 27. Januar stellt zwei hinsichtlich der nationalen Erinnerungsdiskurse diametral verschiedene Fälle einander gegenüber. Vier Merkmale lassen sich für diesen Kontrast anführen: (1) Während Deutschland als Verursacher unmittelbar für den Holocaust Verantwortung trägt, handelt es sich bei Spanien um ein neutrales Land, das am Zweiten Weltkrieg nicht direkt beteiligt war und auch nur einen sehr vermittelten Bezug zum Holocaust besitzt. Gleichwohl handelt es sich historisch betrachtet bei der spanischen Neutralität um ein lediglich formales Merkmal, das zumindest rhetorisch durch die spanische Kriegsbereitschaft sowie die Verbundenheit des Regimes mit den ,Achsenmächten' relativiert werden muss. Auch faktisch griff Spanien in den Zweiten Weltkrieg ein, als General Franco 1941 eine Division Freiwilliger zur Hilfe der Wehrmacht an die Ostfront entsandte (Brinkmann/Peralta Ruiz 2004: 758). Allerdings gibt es keine (signifikante) spanische Beteiligung am Holocaust. (2) Während in Deutschland eine etablierte Gedenkkultur existiert, ist diese in Spanien gerade erst im Entstehen. (3) Das Feld des Gedenkens ist in Deutschland hoch ausdifferenziert und nahezu exklusiv auf den Holocaust bezogen (die Debatten um ein Gedenken an die deutsche Einheit stellen in dieser Hinsicht eine Neuerung dar). In Spanien ist das Gedenken politisch höchst polarisiert und wird zunehmend vermischt mit anderen Erinnerungskulturen, vor allem zum Bürgerkrieg und zur Franco-Diktatur, die in diesem Zuge einen merklichen Aufschwung erleben. (4) Anders als in der weitgehend monolithischen Erinnerungskultur in Deutschland weist das Feld der Erinnerung in Spanien weitreichende Verwerfungen auf, die sich nicht nur im bipolaren Raum der alten Bürgerkriegsparteien von Republikanern und National-Spaniern, sondern zudem auch in den zahlreichen Konflikten zwischen Regionalkulturen (Basken, Katalanen etc.) und Zentralregierung entfalten. 
Der Einbezug Spaniens in die vergleichende Fallstudie ist aus Perspektive der Globalisierung des Holocaustdiskurses von großem Interesse, zumal sich die übergreifende Dynamik der Entwicklung einer transnationalen europäischen Holocausterinnerungskultur besonders an neutralen Ländern zu verdeutlichen scheint. Die während des Weltkriegs eingenommene Haltung wird weniger unter dem nationalen Gesichtspunkt der Interessenbewahrung, sondern mehr unter Berücksichtigung von universellen ethisch-politischen Kriterien wie Menschenrechten, Demokratie und Toleranz bewertet (Françoise 2004). Die in den letzen Jahren stattfindenden gesellschaftlichen Debatten sowie Forschungen, die sich kritisch mit den wirtschaftlichen Beziehungen des Franco-Regimes mit Nazi-Deutschland und einer als restriktiv verurteilten spanischen Flüchtlingspolitik auseinandersetzen, können als Zeichen einer entstehenden Gedächtniskultur in Spanien betrachtet werden, die diese gesamteuropäischen Merkmale aufweist.

Damit sind die Vergleichsdimensionen umrissen. Die Leitfragen für die Untersuchung lassen sich folgendermaßen formulieren: Inwieweit können gemeinsame Merkmale der Erinnerungszeremonien - sofern zu finden - als Ausdruck einer entstehenden transnationalen Erinnerungskultur gedeutet werden? Wie ist ein Erinnern strukturiert, das an einem gleichen Tag stattfindet und des gleichen Ereignisses verschieden gedenkt? Wird in Deutschland der Holocaust in seiner Singularität angesprochen, während in Spanien - wie auch in England - das Ereignis in seiner Beziehung zu anderen Genoziden gesehen wird? (der Tag hat in Spanien nicht denselben Namen, sondern wird als ,Tag des Gedenkens an den Holocaust und für die Verhinderung von Verbrechen gegen die Menschlichkeit‘ begangen). Inwieweit sind die Gedenk-Initiativen eine Folge von Anstrengungen transnationaler Institutionen und politisch-kultureller Eliten? In welchem Verhältnis stehen die offiziellen Gedenkveranstaltungen zu den bürgerschaftlichen und zivilgesellschaftlichen Veranstaltungen, in welchem Ausmaß sind sie von unten, von Bürgerinitiativen und Nachfragen der Zivilgesellschaft geprägt? Welche Muster oder Modelle werden für die symbolische Praxis in den Zeremonien (formensprachliche Elemente, Schlüsselbegriffe, Bilder, Musik usw.) von anderen oder früheren Gedenkkulturen ,importiert'? Gibt es eine Übertragung von religiösen Formen und Deutungsmustern?

\section{Methodisches Vorgehen}

Die Untersuchung richtet sich in der kontrastiven Erforschung der Erinnerungsperformanzen auf zwei miteinander verbundene Ebenen. Im Zentrum stehen (1) videografische Aufzeichnungen der zentralen Gedenkveranstaltungen zum 27. Januar in Deutschland und Spanien sowie (2) fokussierte ethnografische Erhebungen in den Feldern der Erinnerung in beiden Ländern. Für (1) werden videografische Aufzeichnungen der zentralen Gedenkveranstaltungen in beiden Ländern angefertigt, die nachfolgend einer eingehenden Analyse unterzogen werden. Die Analyse widmet sich dabei vor allem der Frage, wie der rituelle Ablauf der beiden Gedenkveranstaltungen aufgebaut ist und realisiert wird. Lassen sich gemeinsame Ritualelemente ausmachen? Worin unterscheiden sich die performativen Ausführungen der beiden Veranstaltungen? Gibt es Spezifika in den beiden Inszenierungen der Gedenkrituale. Sind Konvergenzen zu beobachten? Weisen die jeweiligen Interpretationen der als ähnlich intendierten Vorlage Eigenheiten auf oder dominieren die Ähnlichkeiten - und welche Schlüsse lassen sich daraus für die Frage nach der globalisierten Erinnerungskultur ableiten? Finden eigene Aktivitäten zum 27. Januar statt und welche sind das? In welchem Bezug stehen diese zu den offiziellen Gedenkveranstaltungen? Welche Beteiligungen und Allianzen existieren mit anderen Gruppierungen, Bewegungen und institutionellen Akteuren? Welche Haltung und Beurteilung existieren in der jeweiligen Gruppe gegenüber der Etablierung des 27. Januar und in welchem Verhältnis stehen die Aktivitäten im Vergleich zu den übrigen Gedächtnisaktivitäten? Zur Beantwortung dieser kontextualisierenden Fragen ist (2) eine schrittweise auszudehnende Feldexploration erforderlich, die nach dem Schneeballsystem (bzw. einer iterativzyklischen Forschungslogik, dazu Strübing 2004) Kontakt zu den zentralen Akteuren im Feld der Erinnerung aufnehmen soll. Dabei werden deren Aktivitäten zum 27. Januar erhoben. Expertengespräche und beobachtende Teilnahme ergänzen sich hier wechselseitig.

Konzeptueller und methodologischer Rahmen der Untersuchung ist die Soziologie kommunikativer Gattungen und sozialer Formen (Knoblauch 1995; Günthner/ Knoblauch 1994; Luckmann 2002). Erinnerungsveranstaltungen wie der 27. Januar sind soziale Kommunikationsereignisse, die sich (a) durch unmittelbare Performanz vor einem Präsenzpublikum auszeichnen, mit dem eine Interaktion stattfindet. Außerdem werden sie (b) zusätzlich aufgezeichnet und einem breiteren Publikum medial vermittelt. Als Ereignisse ritueller Kommunikation unter Anwesenden sind sie Gegenstand einer soziologischen Kommunikationsforschung, die sich mit der Verwendung von Sprache und anderen Zeichensystemen in 
Interaktionssituationen beschäftigt. Die soziologische Erforschung natürlicher Kommunikation steht gerade im deutschsprachigen Raum in einem engen Zusammenhang mit der Wissenssoziologie. Sie lässt sich gattungsanalytisch untersuchen.

\section{Erste Analyseerträge}

Einige Vorarbeiten wurden für das Forschungsprojekt schon geleistet: Die Sammlung zentraler Daten wurde aufgenommen und die ersten Analysen am Material durchgeführt, wobei das Potenzial des Materials für eine komparative Analyse geprüft wurde. Insbesondere wurde mit der Analyse der videografischen Aufzeichnungen der spanischen Gedenkveranstaltung vom 27. Januar zwischen 2006 und 2009 begonnen. Der Analysefokus richtet sich dabei auf die zentrale Stelle der Veranstaltung, die im Ritual des Kerzenanzündens kulminiert. Über die letzen Jahre ist eine Entwicklung des Rituals zu beobachten: In Spanien wurde der Gedenkakt zum ersten Mal 2005 zelebriert und hat seit dem weitere vier Male stattgefunden. Dabei können wir eine mehr oder weniger feste Ritualordnung finden, die über diese Zeit nur leicht variiert. Der Akt findet immer im so genannten ,Paraninfo', dem Auditorium der Complutense Universität in Madrid statt. Der Raum befindet sich in einem ehemaligen Jesuitenseminar, das heute für akademische Zeremonien benutzt wird. Die allgemeine rituelle Struktur besteht aus drei alternierenden Elementen: Reden, Gesang und Gebeten. An zentraler Stelle steht der Akt des Kerzenentzündens. Nachdem das Trauerlied der Sinti und Roma „Gelem Gelem“ von einer Gruppe von Sinti-Musikern gespielt und gesungen wird, fängt dieser zentrale Teil des Aktes an. Der Zeremonienmeister, Rektor oder Vizerektor der Universität, verkündet: „Für die sechs Millionen Juden, die in den Konzentrationslagern ermordet wurden" und zugleich erhebt sich ein älterer Mann von seinem Platz, nähert sich den Leuchtern und zündet die erste Kerze an.

Die zweite Kerze wird den „1,5 Millionen in den Gaskammern ermordeten Kindern" gewidmet, die dritte den „in den deutschen KZs Mauthausen, Gusen, Buchenwald und Bergen-Belsen“ ermordeten Spaniern der republikanischen Seite, die im Einverständnis mit Franco nach dem Einmarsch der Wehrmacht in Frankreich als Kriegsgefangene in deutsche KZs verschleppt wurden. Die vierte Kerze dient der Erinnerung der "Sinti und Roma sowie anderer Kollektive, die vom Nazismus verfolgt wurden“. Die fünfte Kerze wird „für die Gerechten unter den Nationen, die ihr Leben für

Abbildung 2. Ritualablauf der Gedenkstunde des Deutschen Bundestags zum Tag des Gedenkens an die Opfer des Nationalsozialismus, 25. Januar 2008, Berlin, Plenarsaal des Bundestages

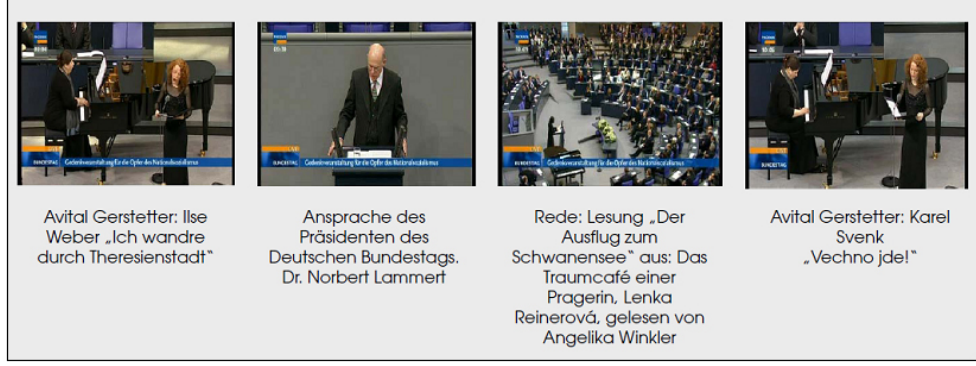

Quelle: eigene Zusammenstellung, TV Material

Abbildung 3. Ritualablauf des ,Día Internacional de la Memoria del Holocausto y de Prevención de los Crímenes contra la Humanidad', 27. Januar 2007, Madrid, Paraninfo der Universität Complutense

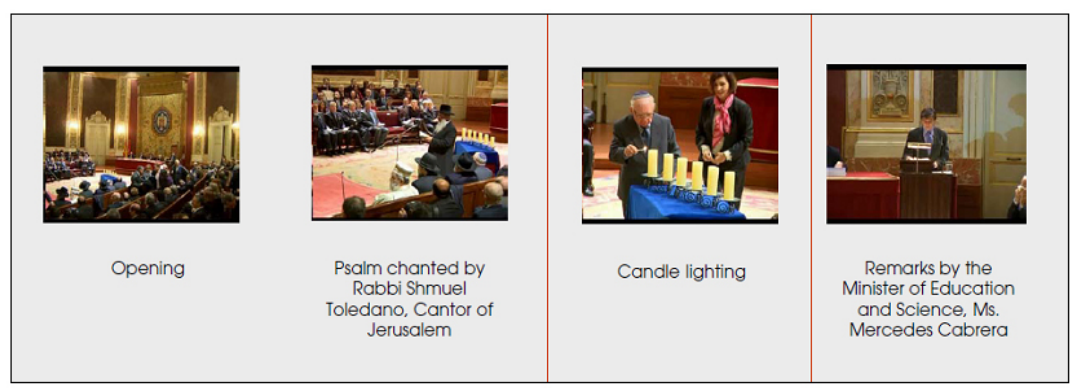

Quelle: eigene Zusammenstellung, Casa Sefarad 
die Rettung der Verfolgten riskiert haben“, entzündet. Die sechste Kerze schließlich ist den „Überlebenden“ gewidmet, „die in Israel eine Zufluchtsstätte fanden“. Die vergleichende Analyse der rituellen Inszenierung dieses Aktes zwischen 2007 und 2009 enthüllt bereits ein bemerkenswertes Merkmal: Das Ritual wird von Jahr zu Jahr zunehmend inklusiver, weil eine wachsende Anzahl an Personen am symbolischen Akt des Kerzenentzündens beteiligt wird. Während 2007 nur sieben Personen damit betraut und namentlich als Stellvertreter des jeweils geehrten Kollektivs hervorgehoben wurden, sind es 2009 bereits 22 Vertreter verschiedener Opferkollektive und anderer Gruppen, die am Kerzenentzünden partizipieren. Das verursacht deutliche Probleme im Ritualablauf.

Die Bedeutung des Aktes erfordert einen kleinen Exkurs zur Symbolik des Kerzenanzündens. Gedenkkerzen (memorial candles) sind eine traditionelle jüdische Form der Erinnerung an die Verstorbenen. Diese werden üblicherweise vier Mal im Jahr während der Yskor-(hebräisch für ,Erinnerung')-Zeremonien in der Synagoge angesteckt. In der dahinter liegenden Bedeutung symbolisiert im Judentum eine Kerzenflamme eine Seele, eine Symbolik, dessen Quelle auf das Buch der Sprüche (Kap. 20, Vers 27) zurückgeht: "die Seele eines Menschen ist das Licht Gottes". Die flackernde Flamme der Gedenkkerze versinnbildlicht die Erinnerung an die Seele der verstorbenen Angehörigen. Die Yom Hashoah-Feiern in den jüdischen Gemeinden beinhalten das Ritual des Kerzenentzündens regelmäßig. Meistens werden dabei sechs Kerzen benutzt, um die Zahl der sechs Millionen im Holocaust ermordeten Juden sinnbildlich darzustellen. In dem an diesem Tag zelebrierten Akt in der israelischen Gedenkstätte Yad Vashem entfachen sechs HolocaustÜberlebende mit jeweils einer Fackel eine Flamme. Die dortige Zeremonie beinhaltet sowohl eine religiöse wie eine nationalzionistische Dimension und wurde 1953 per Gesetz durch den israelischen Staat etabliert (Young 1993). Ungeachtet des religiösen Ursprungs steht dieses Ritual bereits für die Performanz und Darstellung der Erinnerung an Tod und Gewaltschaft und tritt allgemein bei Akten öffentlichen Erinnerns und Betrauerns auch außerhalb religiöser und partikularer Kontexte als allgemeines Massenphänomen auf, etwa beim Tod von Celebrities, am Ground Cero oder nach den Terroranschlägen von London und Madrid.

In unserem Fall zeigt der zentrale Teil der Gedenkveranstaltung - das Entzünden der sechs Kerzen - diesen deutlich jüdischen Ursprung - sowohl durch das Symbol des Yzkor als auch durch die Zahl der Kerzen -, dient jedoch dem darüber hinausweisenden Ziel, die verschiedenen anwesenden Opferkollektive in ein ge- meinsames Gedenken zu integrieren. Es transzendiert den innerjüdischen Bezug und wird zum inklusiven Ritual, indem es die spanische Beziehung zum Holocaust durch die Beteiligung von republikanischen KZÜberlebenden sowie Sinti und Roma betont. Zudem werden universale Kategorien angesprochen (Kinder, ,Gerechte', Überlebende), was das Event der Tendenz und der Intention nach transformiert: Die jüdische Shoah der jüdischen Zeremonie wird zum universellen Holocaust. Mit diesem umfassenderen Begriff werden nicht nur die jüdischen Opfer des Genozids erinnert und geehrt, sondern alle Verfolgten des Nazi-Regimes (Sinti und Roma sowie politisch Verfolgte werden symbolisch mit einer Kerze und einem Vertreter, der sie anzündet, eingeschlossen). Andere Gruppen wie z.B. Zeugen Jehovas oder Homosexuelle werden zwar nicht explizit erwähnt, sind doch zumindest implizit in die Formulierung "Sinti und Roma und andere von Nazismus verfolgte Kollektive“ eingeschlossen. Insgesamt verweisen diese ersten Analysen, die weiterer Verfeinerung bedürfen, auf interessante Erkenntnisse für die vermutete Tendenz in Richtung auf eine Universalisierung wie auch eine Popularisierung von religiösen Symbolen und Konzepten in modernen Erinnerungsritualen. Es ist bei dieser ersten Datenauswertung außerdem hervorzuheben, dass dieser zentrale Akt mit den Kerzen eine klare Botschaft der Affirmation und Positivität ausdrückt.

\section{SCHLUSS}

Im Zuge der Einrichtung des 27. Januars als, Internationalem Holocaust-Gedenktag' bildet sich schrittweise die Etablierung einer in verschiedenen Kulturen gleichzeitig vollzogenen, öffentlich performierten Erinnerung an den Holocaust heraus, welche - unbeschadet manifester Absichten - Grenzen zwischen den Kollektiven von Opfern, Tätern und Unbeteiligten zunehmend in Frage zu stellen scheint. Zumindest treten Universalisierungsbemühungen in ein paradoxes Verhältnis zu historischen Singularitätsansprüchen. In dieser der Absicht nach global und damit ,kulturübergreifend' orientierten performativen Gedächtniskultur werden aber gerade die rituell zum Ausdruck gebrachten partikularen Anliegen der einzelnen (,kulturellen') Kollektive der Gefahr einer symbolischen Verwischung ausgesetzt. Mit unserer vergleichenden Untersuchung von Gedenkveranstaltungen in Deutschland und Spanien explorieren wir anhand dieses Falles videoanalytisch und ethnografisch diese gegenwärtige Ritualform im Spannungsfeld zwischen Globalisierung (des Gedächtnisses) und öffentlicher Präsentation kultureller Eigenart. 
1 http://www.holocausttaskforce.org/ about-the-itf/stockholm-declaration. html?lang=de

\section{BIBLIOGRAFÍA}

Alexander, Jeffrey (2002). On the Socia Construction of Moral Universals. The 'Holocaust' from War Crime to Trauma Drama. In: European Journal of Social Theory 5(1): 5-85

Assmann, Jan (1997). Das kulturelle Gedächtnis. Schrift Erinnerung und politische Identität in frühen Hochkulturen. München: C.H. Beck.

Baer, Alejandro (2005). El testimonio audiovisual. Imagen y memoria de Holocausto. Madrid: Centro de Investigaciones Sociológicas, $\mathrm{CIS}$.

Biersack, Aletta (Hrsg.) (1991). Clio in Oceania. Toward a Historical Anthropology. Washington: Smithsonian Institution Press.

Brinkmann, Sören/Peralta Ruiz, Víctor (2004). Spanien. Weder Täter noch Opfer? Mythen der Nationen. In: Flacke, Monika (2004): 757-772.

Buser, Tina/Rauer, Valentin (2004). Gianfranco Finis Erinnerungspolitik. Eine Medienanalyse zu den Gedenkbesuchen in den Fosse Ardeatine und in Auschwitz. In: Giesen, Bernhard/Schneider, Christoph (2004): 239-268.

Connerton, Paul (1989). How societies remember. Cambridge: Cambridge University Press.

Dubiel, Helmut (2003). The Remembrance of the Holocaust as a Catalyst for a Transnational Ethic. New German Critique 90: 59-70.

Flacke, Monika (Hrsg.) (2004). Mythen der Nationen. 1945 - Arena der Erinnerungen. Katalog zur Ausstellung im Deutschen Historischen Museum Berlin. Mainz: Phillip von Zabern.

Françoise, Etienne (2004). Meistererzählungen und Dammbrüche. Die Erinnerung an den Zweiten Weltkrieg zwischen $\mathrm{Na}$ tionalisierung und Universalisierung. In: Flacke, Monika (2004): 13-28.

Giesen, Bernhard (2004). Triumph and Trauma. Boulder CO: Paradigm Publishers.

Giesen, Bernhard/Schneider, Christoph (Hrsg.) (2004). Tätertrauma. Nationale
Erinnerungen im öffentlichen Diskurs. Konstanz: UVK.

Gillis, John R. (1994). Commemorations. The Politics of National Identity. New Jersey: Princeton University Press.

Goffman, Erving (1983). The Interaction Order. In: American Sociological Review 48(1): 1-17.

Günthner, Susanne/Knoblauch, Hubert (1994). Forms are the food of faith. Gattungen als Muster kommunikativen Handelns. In: Kölner Zeitschrift für Soziologie und Sozialpsychologie 46(4): 693-723.

Knoblauch, Hubert (1995). Kommunikationskultur. Die kommunikative Konstruktion kultureller Kontexte. Berlin: Walter de Gruyter.

Leggewie, Claus (2009). Schlachtfeld Europa. In: Blätter für deutsche und internationale Politik 2: 81-93.

Le Goff, Jacques/Nora, Pierre (Hrsg.) (1974). Faire de l'histoire. Vol. I: Nouveaux problèmes. Paris: Gallimard.

Lenz, Claudia/von Wrochem, Oliver/ Schmidt, Jens (Hrsg.) (2002). Erinnerungskulturen im Dialog. Europäische Perspektiven auf die NS-Vergangenheit. Berlin: Unrast.

Levy, Daniel/Sznaider, Natan (2001). Erinnerung im globalen Zeitalter. Der Holocaust. Frankfurt/M.: Suhrkamp.

Luckmann, Thomas (2002). Zur Methodologie (mündlicher) kommunikativer Gattungen. In: Luckmann, Thomas (2002): 183-200.

Luckmann, Thomas (2002). Wissen und Gesellschaft. Ausgewählte Aufsätze 19812002. Konstanz: UVK.

MacAlloon, John J. (2009). Genre and Risk in Olympic Ceremonies. In: Schlesier Renate/Zellmann, Ulrike (2009): 31-52.

Nora, Pierre (1974). Le retour de l'événement. In: Le Goff, Jacques/Nora, Pierre (1974): 210-229.

$\emptyset$ stergård, Uffe (2008). Der Holocaust und europäische Werte. In: Aus Politik und
Zeitgeschichte 1(31.12.2007). Online verfügbar unter: http://www.bundestag.de/dasparlament/2008/01-02/Beilage/005.html (Stand: 24.11.2009).

Probst, Lothar (2002). Europäisierung des Holocaust. Eine neue Zivilreligion für Europa? In: Kommune 7: 42-46.

Sahlins, Marshall (1991). The Return of the Event, Again. In: Biersack, Aletta (1991): 37-99.

Schlesier, Renate/Zellmann, Ulrike (Hrsg.) (2009). Ritual als provoziertes Risiko. Würzburg: Könighausen \& Neumann.

Soeffner, Hans-Georg (2000). Auf dem Rükken eines Tigers. Über die Hoffnung, Kollektivrituale als Ordnungsmächte in interkulturellen Gesellschaften kultivieren zu können. In: Soeffner, Hans-Georg (2000): 254-279.

Soeffner, Hans-Georg (2000). Gesellschaft ohne Baldachin. Über die Labilität von Ordnungskonstruktionen. Weilerswist: Velbrück Wissenschaft.

Soeffner, Hans-Georg (2006). Selbsterlösung. Einige Grundzüge deutscher Erinnerungspolitiken. In: Soeffner, HansGeorg (2006): 103-128.

Soeffner, Hans-Georg (2006). Zeitbilder. Versuche über Glück, Lebensstil, Gewalt und Schuld. Frankfurt/M./New York: Campus.

Strübing, Jörg (2004). Grounded Theory. Zur sozialtheoretischen und epistemologischen Fundierung des Verfahrens der empirisch begründeten Theoriebildung. Wiesbaden: VS Verlag.

Traverso, Enzo (2007). Gebrauchsanweisungen für die Vergangenheit. Geschichte, Erinnerung, Politik. Münster: Unrast.

Young, James E. (1993). The Texture of Memory. Holocaust Memorials and Meaning. New Haven/London: Yale University Press.

Zifonun, Dariuš (2004). Gedenken und Identität. Der deutsche Erinnerungsdiskurs. Frankfurt/M.: Campus. 